\title{
Screening for Neurocognitive Impairment in HIV Individuals: The Utility of the Montreal Cognitive Assessment Test
}

Rodrigo Hasbun ${ }^{1},{ }^{*}$, Jairo Eraso ${ }^{1}$, Sweeya Ramireddy ${ }^{1}$, D’ Arcy Wainwright ${ }^{1}$, Lucrecia Salazar ${ }^{1}$, Richard Grimes ${ }^{1}$, Michele York ${ }^{2}$ and Adriana Strutt ${ }^{2}$

${ }^{1}$ Department of Internal Medicine, University of Texas Health Science Center in Houston, USA

${ }^{2}$ Department of Neurology, Division of Neuropsychology, Baylor College of Medicine, Houston, USA

\section{Background}

Human Immunodeficiency virus (HIV) associated neurocognitive disorders have been reported in up to $50 \%$ of patients on highly active antiretroviral therapy (HAART) [1]. Even though screening for neurocognitive impairment $(\mathrm{NCI})$ is not routinely done or recommended by current treatment guidelines [2], NCI is associated with HAARTnoncompliance and functional impairment [3], and is currently being linked to cerebrospinal fluid escape (positive CSF HIV RNA PCR in the setting of undetectable serum HIV RNA PCR) [4]. The Montreal cognitive assessment (MoCA) has been validated and widely used in several countries and languages in non-HIV infected populations to screen for cognitive impairment [5,6]. There is very limited data in HIV positive patients [7], a population that is associated with high rates of co-existing infections and comorbidities including depression and drug abuse. The purpose of our study was to evaluate the utility of the MoCA as a screening tool for $\mathrm{NCI}$ in HIV infected individuals.

\section{Study design}

Prospective observational study of adult ( $>17 y$ rs of age) ART-naïve HIV-1-infected consecutive individuals presenting to Thomas Street Health Center (TSHC) in Houston, TX, between 8/24/10 to 09/27/11 for their baseline evaluation. Patients were excluded if they had prior ART experience or refused to participate. The study coordinators screened and enrolled the patients while waiting to be seen by the TSHC triage nurse or by the physician. The study was approved by the University of Texas Committee for the protection of human subjects and by the Harris County Hospital District Research committee. All subjects signed an informed consent.

Baseline sociodemographic information was collected and patients were screened for the following co-existing conditions: syphilis (RPR), active drug use (urine drug screen), Hepatitis B and C and Toxoplasma gondii (serologies), tuberculosis exposure (PPD test), and depression by using the Beck Depression Inventory-II [8].

All patients underwent screening for NCI using the MoCA. This screening tool assesses eight cognitive domains (attention and concentration, executive functions, memory, language, visuoconstructional skills, conceptual thinking, calculations, and orientation), which are scored within a range of 0-30 points (higher scores reflecting better cognitive function). This rapid screen takes approximately 10 minutes to administer and is available in 30 languages. A score of less than 26 is considered abnormal $[5,6]$.

In order to diagnose cognitive impairment, an additional battery of neuropsychological testing were performed which included the Symbol digit modalities test [9] Stroop color and Word test [10] Wechsler adult intellegence-III subtests of Picture Completion [11] Matrix reasoning and Block design [11] and Vocabulary QQ [12] to evaluate various cognitive domains including attention/working memory; speed of information processing inhibition, language; and visuospatial construction. The screening instrument and the battery of neuropsychological tests were chosen based on several criteria: 1) valid and sensitive measures to assess the HIV associated subcortical neurocognitive dysfunction, 2) availablilty in English and Spanish versions, and 3) limited administration time to reduce the burden on the patients. Neurocognitive impairment (NCI) was defined as greater than 1.5 standard deviation below the mean on 2 or more neuropsychological measures. We decided to use this higher cut-off to prevent overestimating the real prevalence of $\mathrm{NCI}$ as recently suggested by Gisslen et al. [13]. We did not assess the prevalence of HIV-associated neurocognitive disorders because we did not evaluate the impact on activities of daily living [14].

\section{Statistical analyses}

The first step in the analysis was a comprehensive description of the patient's baseline sociodemographic factors, HIV risk factor profile, co-existing comorbidities including depression and active drug abuse, and serum HIV viral load, CD4 levels, and prevalence of cognitive impairment. Bivariate analysis were then conducted by using the Pearson's $\mathrm{X}^{2}$ test or Fisher's exact test to identify factors that were significantly associated with neurocognitive impairment $(\mathrm{P}<0.05)$. All clinically cogent baseline variables with a $\mathrm{P}$ value $<0.20$ were entered into a logistic regression model to identify variables independently associated with NCI. The diagnostic accuracy of the MoCA was tested by calculating the sensitivity, specificity, negative predictive value, and the positive predictive value. All statistical analyses were conducted using SPSS version 19 (SPSS).

\section{Results}

After screening a total of 118 patients, 11 patients were excluded because they refused participation in the study and 7 patients because they had prior HAART. A total of $100 \mathrm{HIV}$ positive ART naïve patients were enrolled (Table 1). The median was 43 years (22-64); the majority were male (75\%), African American (71\%), unemployed (50\%) and two-thirds had less than 12 years of education. One-third of cases (31\%) had an AIDS diagnosis and a high baseline serum HIV viral load (>100,000 copies $/ \mathrm{ml})$. Co-infections were common, including hepatitis B (30\%); syphilis (22\%) and hepatitis C (15\%). Active drug use was seen in $37 \%$ of patients and $41 \%$ of the patients had moderate to severe depression. Only one patient used the Spanish versions of the neurocognitive tests.

*Corresponding author: Rodrigo Hasbun, Department of Internal Medicine, University of Texas Health Science Center in Houston, 6431 Fannin St. 2.112 MSB, Houston, Texas 77030, Tel: (713) 500-7140; Fax (713) 500-5495; E-mail: Rodrigo.Hasbun@uth.tmc.edu

Received October 25, 2012; Accepted November 23, 2012; Published November 26, 2012

Citation: Krol M, Balcerowski A, Luczynska M, Szkudlarek U, Nowak D (2012) Screening for Neurocognitive Impairment in HIV Individuals: The Utility of the Montreal Cognitive Assessment Test. J AIDS Clinic Res 3:186. doi:10.4172/21556113.1000186

Copyright: ( $\odot 2012$ Hasbun R, et al. This is an open-access article distributed under the terms of the Creative Commons Attribution License, which permits unrestricted use, distribution, and reproduction in any medium, provided the original author and source are credited. 
Citation: Hasbun R, Eraso J, Ramireddy S, Wainwright D’A, Salazar L et al. (2012) Screening for Neurocognitive Impairment in HIV Individuals: The Utility of the Montreal Cognitive Assessment Test. J AIDS Clinic Res 3:186. doi:10.4172/2155-6113.1000186

Page 2 of 3

\begin{tabular}{|c|c|c|c|c|}
\hline Variables & $\begin{array}{c}\text { Total } \\
(n=100)\end{array}$ & $\begin{array}{c}\text { No cognitive } \\
\text { impairment } \\
(n=25)\end{array}$ & $\begin{array}{c}\text { Cognitive } \\
\text { impairment }{ }^{\text {a }} \\
(n=75)\end{array}$ & $P$ value \\
\hline Age $>50$ years & 18 & 6 & 12 & 0.36 \\
\hline Male sex & 75 & 21 & 54 & 0.23 \\
\hline African-American race & 71 & 16 & 55 & 0.44 \\
\hline Education $\leq 12$ years & 68 & 13 & 55 & 0.08 \\
\hline Unemployed & 50 & 16 & 34 & 0.16 \\
\hline CD4 nadir $<200^{b}$ & 32 & 9 & 23 & 0.39 \\
\hline $\begin{array}{l}\text { HIV viral load } \\
\geq 100,000 \text { copies } / \mathrm{ml}\end{array}$ & 31 & 8 & 23 & 0.54 \\
\hline Homosexual risk factor & 45 & 7 & 38 & 0.06 \\
\hline RPR Positive & 22 & 5 & 17 & 0.51 \\
\hline Hepatitis B seropositive & 30 & 11 & 19 & 0.08 \\
\hline $\begin{array}{l}\text { Hepatitis C } \\
\text { seropositive }\end{array}$ & 15 & 2 & 13 & 0.34 \\
\hline Urine drug screen positive $\mathrm{c}$ & 38 & 9 & 29 & 0.81 \\
\hline Moderate/severe depression d & 41 & 7 & 34 & 0.16 \\
\hline MoCA score $<26^{e}$ & 79 & 15 & 64 & 0.01 \\
\hline
\end{tabular}

a defined as greater than 1.5 standard deviation below the mean on 2 or more neuropsychological measures;

${ }^{\mathrm{b}} \mathrm{CD} 4<200$ at initial evaluation;

c cannabinoids (22), cocaine (11), opiates (11), amphetamines (3), PCP (2),

benzodiazepines (2), polysubstance abuse (13);

'Beck's Depression Inventory score;

e diagnostic accuracy of the MoCA: sensitivity: $85 \%$; specificity: $40 \%$; negative predictive value: $48 \%$; positive predictive value: $81 \%$.

Table 1: Bivariate analysis of variables associated with neurocognitive impairment in 100 HIV antiretroviral therapy naïve individuals.

NCI was detected in $75 \%$ of patients. On bivariate analysis, only a MoCA score $<26$ was associated with NCI (OR 5.55 (2.08-14.78)) and had good screening accuracy (sensitivity: $85 \%$; specificity: $40 \%$; negative predictive value: $48 \%$; positive predictive value: $81 \%$ ) (Table 1). After adjusting for education level, depression, employment status, HIV risk behavior and coexisting hepatitis $\mathrm{B}$, the MoCA was still associated with $\mathrm{NCI}$ in the logistic model (OR $4.83(1.70-12.74))(\mathrm{P}=0.003)$.

\section{Discussion}

Cognitive impairment in HIV infected individuals is associated with HAART-adherence and has functional consequences [3]. Despite its high prevalence in HIV, screening for neurocognitive impairment is problematic. Assessing symptoms with standardized questionaires (e.g., medical outcomes survey) only identifies symptomatic disease and may not be predictive of objective performance [7]. The most commonly studied screening tools that have been used and validated in HIV associated neurocognitive disorders include three types of dementia scales and the Mini-mental status examination (MMSE) [7]. The MMSE is quick to administer and many clinicians are familiar with its use but it has poor sensitivity and specificity for HIV-associated neurocognitive disorders (HAND). The HIV-associated dementia (HAD) scale tests memory recall, registration, psychomotor speed and attention and it is useful in diagnosing HAD. In the HAART era though, the majority of the neurocognitive disorders are mild in severity and the sensitivity of the HAD scale is low. The sensitivity of the HAD scale can be improved by applying demographically adjusted normative standards [15].

The MoCA is a widely used and available test that screens for mild cognitive impairment in HIV-uninfected individuals with better sensivitivity that the Mini-mental status examination [7]. The MoCA is a one-page test that is administered in 10 minutes and can be used to track changes in cognitive ability over time. The MoCA is also an attractive screening tool because it is free and tests multiple domains of function. To our knowledge, there are only a few studies evaluating the use of the MoCA

in HIV-infected patients [7]. In a study by Koski et al. [16], the MoCA was found to measure cognitive ability in 75 patients by using computerized tasks but it had a poorer precision in patients with higher cognitive ability. It was also noted that supplementation with seven tasks assessing frontal lobe function resulted in a better psychometric assessment. Overton et al. [17] reported the use of the MoCA in 119 HIV-infected individuals with a prevalence of HAND of $64 \%$. The MoCA had a sensitivity of 59\% and a specificity of $81 \%$ by using a MoCA cutoff of $<26$; the sensitivity increased to $83 \%$ if the cut-off was $<27$. Chan et al. [18] studied the utilized the MoCA in 132 HIV positive adults from Singapore to diagnose HAND. To our knowledge, there is no study evaluating the MoCA in a cohort of HAART naive individuals.

The prevalence of neurocognitive impairment was in $75 \%$ of our ART naïve patient population with high rates of unemployment, active drug use, depression and concomitant comorbidities. The only predictor of NCI in our study was a MoCA score <26. In this setting, the MoCA had a moderate diagnostic accuracy with sensitivity: $85 \%$; specificity: $40 \%$; negative predictive value: $48 \%$; positive predictive value: $81 \%$ (Table 1 ). The negative and positive predictive values would differ in other patient populations with different degree of NCI prevalence.

Our study had strengths and limititations. First, to our knowledge this is the first prospective evaluation of the MoCA in HIV-ART naïve individuals. Second, all patients were screened for active drug use, depression and other comorbidities that can affect cognition. Third, all patients underwent a complete neuropsychological battery of tests to determine if they had neurocognitive impairment. The main limitation of the study was the high prevalence of active drug use, depression, unemployment, low education level and associated comorbidities that can also affect cognition. This is an accurate representation of the indigent population that we serve at Thomas Street Health Science Center in Houston and as such this result can not be generalized to other patient populations without further studies. In addition, functional impairment was not assessed so we could not make the distinction between the two HIV-associated neurocognitiive disorders (asymptomatic neurocognitive impairment and mild neurocognitive disorder) [14].

In summary, the MoCA is a quick and quantitative tool that can be used to screen for neurocognitive impairment in HIV-positive patients with high sensitivity. Patients with HIV and a MoCA score $<26$ should undergo further neuropsychological testing to assess cognitive impairment.

\section{Acknowledgement}

This study was supported by the Glaxo Smith Kline HIV CIRA award (PI Hasbun), and by the Baylor-UT Houston Center for AIDS research core support grant number 5P30AI036211-17 from the National Institute of Allergy and Infectious Diseases.

\section{References}

1. Ellis RJ, Badiee J, Vaida F, Letendre S, Heaton RK, et al. (2011) CD4 nadir is a predictor of HIV neurocognitive impairment in the era of combination antiretroviral therapy. AIDS 25: 1747-1751

2. Panel on Antiretroviral Guidelines for Adults and Adolescents. Guidelines for the use of antiretroviral agents in HIV-1-infected adults and adolescents. Dept of Health and Human Services.

3. Gorman AA, Foley JM, Ettenhofer ML, Hinkin CH, van Gorp WG (2009) Functional consequences of HIV-associated neuropsychological impairment Neuropsychol Rev 19: 186-203.

4. Valcour VG (2011) Evaluating cognitive impairment in the clinical setting: practical screening and assessment tools. Top Antivir Med 19: 175-180.

5. Nasreddine ZS, Phillips NA, Bédirian V, Charbonneau S, Whitehead V, et al (2005) The Montreal Cognitive Assessment, MoCA: a brief screening tool for mild cognitive impairment. J Am Geriatr Soc 53: 695-699. 
Citation: Hasbun R, Eraso J, Ramireddy S, Wainwright D'A, Salazar L et al. (2012) Screening for Neurocognitive Impairment in HIV Individuals: The Utility of the Montreal Cognitive Assessment Test. J AIDS Clinic Res 3:186. doi:10.4172/2155-6113.1000186

6. McLennan SN, Mathias JL, Brennan LC, Stewart S (2011) Validity of the montreal cognitive assessment (MoCA) as a screening test for mild cognitive impairment $(\mathrm{MCl})$ in a cardiovascular population. J Geriatr Psychiatry Neurol 24: $33-38$

7. Valcour V, Paul R, Chiao S, Wendelken LA, Miller B (2011) Screening for cognitive impairment in human immunodeficiency virus. Clin Infect Dis 53: 836842.

8. Vanheule S, Desmet M, Groenvynck H, Rosseel Y, Fontaine J (2008) The factor structure of the Beck Depression Inventory-II: an evaluation. Assessment 15: 177-187.

9. Tamayo F, Casals-Coll M, Sánchez-Benavides G, Quintana M, Manero RM, et al. (2012) Spanish normative studies in a young adult population (NEURONORMA young adults Project): norms for the verbal span, visuospatial span, LetterNumber Sequencing, Trail Making Test and Symbol Digit Modalities Test. Neurologia 27: 319-329.

10. Gunner JH, Miele AS, Lynch JK, McCaffrey RJ (2012) Performance of nonneurological older adults on the Wisconsin Card Sorting Test and the Stroop Color-Word Test: normal variability or cognitive impairment? Arch Clin Neuropsychol 27: 398-405.

11. Wisdom NM, Mignogna J, Collins RL (2012) Variability in Wechsler Adult Intelligence Scale-IV subtest performance across age. Arch Clin Neuropsychol 27: 389-397.

12. Al-Ghatani AM, Obonsawin MC, Binshaig BA, Al-Moutaery KR (2011) Saudi normative data for the Wisconsin Card Sorting test, Stroop test, Test of Non-verbal Intelligence-3, Picture Completion and Vocabulary (subtest of the Wechsler Adult Intelligence Scale-Revised). Neurosciences (Riyadh) 16: 29-41.

13. Gisslén M, Price RW, Nilsson S (2011) The definition of HIV-associated neurocognitive disorders: are we overestimating the real prevalence? BMC Infect Dis 11: 356.

14. Antinori A, Arendt G, Becker JT, Brew BJ, Byrd DA, et al. (2007) Updated research nosology for HIV-associated neurocognitive disorders. Neurology 69 : 1789-1799.

15. Morgan EE, Woods SP, Scott JC, Childers M, Beck JM, et al. (2008) Predictive validity of demographically adjusted normative standards for the HIV Dementia Scale. J Clin Exp Neuropsychol 30: 83-90.

16. Koski L, Brouillette MJ, Lalonde R, Hello B, Wong E, et al. (2011) Computerized testing augments pencil-and-paper tasks in measuring HIV-associated mild cognitive impairment( $\left.{ }^{*}\right)$. HIV Med 12: 472-480.

17. Overton E, Ances B, Grubb J, Parker N, Spitz T, et al. (2011) Novel screening tools for HIV-associated neurocognitive disorders. 18th Conference on Retroviruses and Infections (CROI), Washington Univ in St Louis, Sch of Med, $\mathrm{MO}, \mathrm{US}$.

18. Chan LG, Kandiah N, Chua A (2012) HIV-associated neurocognitive disorders (HAND) in a South Asian population-contextual application of the 2007 criteria. BMJ Open 2: e000662. 